\title{
Electrical transport through a single-electron transistor strongly coupled to an oscillator
}

\author{
C. B. Doiron, ${ }^{1}$ W. Belzig, ${ }^{2}$ and C. Bruder ${ }^{1}$ \\ ${ }^{1}$ Department of Physics and Astronomy, University of Basel, CH-4056 Basel, Switzerland \\ ${ }^{2}$ University of Konstanz, Department of Physics, D-78457 Konstanz, Germany \\ (Received 11 August 2006; revised manuscript received 11 October 2006; published 30 November 2006)

\begin{abstract}
We investigate electrical transport through a single-electron transistor coupled to a nanomechanical oscillator. Using a combination of a master-equation approach and a numerical Monte Carlo method, we calculate the average current and the current noise in the strong-coupling regime, studying deviations from previously derived analytic results valid in the limit of weak coupling. After generalizing the weak-coupling theory to enable the calculation of higher cumulants of the current, we use our numerical approach to study how the third cumulant is affected in the strong-coupling regime. In this case, we find an interesting crossover between a weak-coupling transport regime where the third cumulant heavily depends on the frequency of the oscillator to one where it becomes practically independent of this parameter. Finally, we study the spectrum of the transport noise and show that the two peaks found in the weak-coupling limit merge on increasing the coupling strength. Our calculation of the frequency dependence of the noise also allows one to describe how transport-induced damping of the mechanical oscillations is affected in the strong-coupling regime.
\end{abstract}

DOI: 10.1103/PhysRevB.74.205336

PACS number(s): 73.23.- b, 85.85.+j, 72.70.+m, 73.23.Hk

\section{INTRODUCTION}

Nanoelectromechanical devices-i.e., nanostructures in which electric transport through a device is influenced by its mechanical degrees of freedom and vice versa-have attracted a lot of interest recently. ${ }^{1,2}$ On the one hand, these devices are promising for applications like sensors or ultrasensitive mass detectors. On the other hand, they have opened up new directions in fundamental research, with projects to cool nanomechanical systems to the quantum limit. ${ }^{3,4}$

The nanomechanical properties of single-electron transistors (SET's) are of particular interest in this context. The central island of a SET may be allowed to mechanically move between the two leads, such that electrons can tunnel on the island if the island has approached one lead and leave it again once it has mechanically moved to the other lead. These "shuttles" have been investigated in great detail. ${ }^{5-13}$ Another possibility to couple the electrical and mechanical properties of the device is to design the SET such that its capacitive coupling to the gate depends on the displacement of a mechanical oscillator. Thus, mechanical degrees of freedom of the system may strongly influence the currentvoltage characteristics, current noise, and higher cumulants of the current. ${ }^{14-20}$ It has been shown that the Coulombblockade peaks are split for harmonic oscillations and are broadened by thermal oscillations. Knowledge of the SET transport properties therefore allows one to determine the characteristics of the oscillator, such as its amplitude and frequency. In such systems, electron tunneling through the island also has an effect on the motion of the oscillator. This back action leads to fluctuations in the oscillator position and to damping. ${ }^{21}$

Practical implementations of oscillator-coupled SET's can be realized by combining nanostructured silicon resonators with metallic SET's. ${ }^{22,23}$ Another possibility is to build SET's from suspended carbon nanotubes that act as quantum dots. ${ }^{24}$ Quite recently, mechanical oscillations of the nanotube in such a device have been directly observed. ${ }^{25}$
In the following, we will consider a SET coupled to a classical harmonic oscillator. This system has already been studied extensively. ${ }^{14,15}$ However, previous studies investigated the regime where the coupling between the oscillator and the SET is weak and the question as to what happens when the coupling is increased is still of great theoretical interest, ${ }^{26}$ even if this regime is not readily accessible in the current generation of experiments. In this article, we will use a combination of a master-equation approach and a numerical Monte Carlo procedure to calculate the electrical current and its second and third cumulants and study how they are modified by coupling to the oscillator, in the regime where the coupling is strong. We will also study the frequency dependence of the transport noise.

The paper is organized as follows: in Sec. II, we discuss the system and model whose strong-coupling limit will be studied in the subsequent sections. The model and masterequation approach that we use follow closely Ref. 14. This section also introduces the important dimensionless coupling parameter $\kappa$, which is the ratio of the typical mechanical energy scale and the source-drain voltage. In the next section, Sec. III, we calculate the probability distributions of the position of the oscillator if the SET is in state $N$ or $N+1$ using a numerical Monte Carlo procedure. The Gaussian form predicted by the weak-coupling approach is modified dramatically in the strong-coupling regime. In Sec. IV, we calculate the average current through the device at the charge-degeneracy point of the SET at which the current is maximal and discuss the deviations from the weak-coupling results. Finally, in Secs. V and VI we extend our studies to the current noise and the third cumulant of the current.

\section{COUPLED SET-OSCILLATOR SYSTEM DESCRIPTION}

To describe the coupled SET-nanomechanical-oscillator system, we use the formalism introduced in Ref. 14.

The system that we consider is shown in Fig. 1 in a schematic way and consists of two symmetric tunnel junctions, 


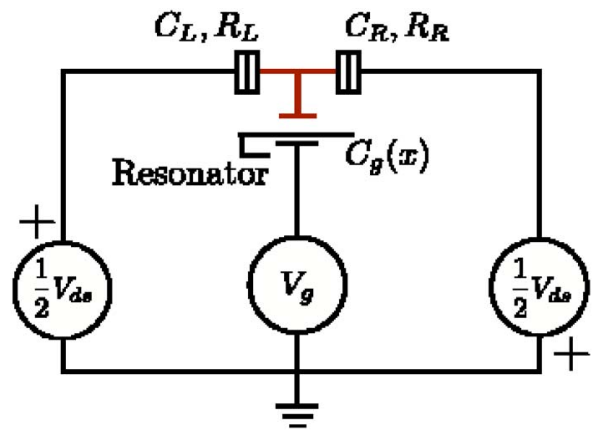

FIG. 1. (Color online) Circuit diagram of the system studied. The gate capacitance of the SET depends on the displacement of a mechanical oscillator, leading to a coupling of the electrical transport through the device and the mechanical motion of the oscillator.

each with resistance $R$ and capacitance $C$, connected in series. Transport through the SET is described using the orthodox model, where only two charge states are considered and where the current arises only from sequential tunneling. ${ }^{27,28}$ In this case, transport is governed by four tunneling rates $\Gamma_{i}^{\sigma}$ where $i=R, L$ is the lead index and $\sigma=+,-$ indicates the direction of the tunneling. In this work, we adopt the convention that the forward $(+)$ direction, given by the polarity of the bias voltage, is from the right to the left lead. The tunneling rates can be calculated using Fermi's golden rule and are a function of the difference in free energy $\Delta E$ of the system before and after a tunneling event:

$$
\Gamma_{i}^{\sigma}=\frac{1}{\operatorname{Re}^{2}} \Delta E_{i}^{\sigma} f\left(\Delta E_{i}^{\sigma}\right),
$$

where $f(x)=\left(1-e^{-x / k_{B} T}\right)^{-1}$, with $T$ the electronic temperature. The energy differences $\Delta E_{i}^{\sigma}$ are given by

$$
\begin{aligned}
& \Delta E_{L}^{+}=-\Delta E_{L}^{-}=e V_{d s}\left(\frac{1}{2}+\left(2 N-2 N_{g}+1\right) \frac{E_{c}}{e V_{d s}}\right), \\
& \Delta E_{R}^{+}=-\Delta E_{R}^{-}=e V_{d s}\left(\frac{1}{2}-\left(2 N-2 N_{g}+1\right) \frac{E_{c}}{e V_{d s}}\right),
\end{aligned}
$$

where $V_{d s}$ is the applied drain-source voltage, $E_{c}=e^{2} /(2 C$ $\left.+C_{g}\right)$ is the charging energy of the island, and $N_{g}=C_{g} V_{g} / e$ is the optimal number of charges on the island. Knowing the different rates, the average current $I$ flowing through the SET can be calculated using

$$
\text { I/e }=P_{N+1} \Gamma_{L}^{+}-P_{N} \Gamma_{L}^{-}=P_{N} \Gamma_{R}^{+}-P_{N+1} \Gamma_{R}^{-},
$$

where $P_{N(N+1)}$ is the probability to find the island in charge state $N(N+1)$ in the stationary limit.

Our model of the SET remains valid as long as its charging energy $E_{c}$ is large compared to the electronic thermal energy $k_{B} T_{e}$ and the source-drain bias $e V_{d s}$. We will neglect second-order tunneling processes (cotunneling).

In this work, the nanomechanical oscillator is modeled as a single, classical, harmonic oscillator of frequency $\omega_{0}$. Introducing a time scale $\tau_{t}=\operatorname{Re} / V_{d s}$ which has the physical meaning of an average time between tunneling events, we can use a dimensionless parameter

$$
\epsilon=\omega_{0} \tau_{t}=\omega_{0} \frac{R e}{V_{d s}}
$$

to compare the typical electrical and mechanical time scales.

A particular state of the oscillator is then represented by a position $x$ and velocity $u$. We choose $x=0$ to be the equilibrium point of the oscillator when $N$ charges are on the SET. When the charge state of the island is changed-for example, from $N$ to $N+1$ - the change in the electrostatic forces between the oscillator (kept at constant potential $V_{g}$ ) and the SET effectively shifts the equilibrium position of the resonator. The distance between the equilibrium positions when $N$ and $N+1$ charges are on the island defines a natural length scale $x_{0}$ of the problem, $x_{0}=-2 E_{c} N_{g} /\left(m \omega_{0}^{2} d\right)$. Here, $d$ is the distance separating the oscillator's equilibrium position and the SET island, such that the gate capacitance depends on $x$ like $C_{g}(x) \sim(d+x)^{-1} \sim 1-x / d$. From now on, we will also use dimensionless rates; i.e., all the rates will be given in units of $\tau_{t}^{-1}$.

Coupling a SET and a nanomechanical oscillator system is readily done by using the oscillator itself as the SET's gate. In this configuration, the capacitive coupling between the oscillator and the SET depends on the distance between them and by extension on the oscillator's position, effectively allowing one to monitor the dynamics of the oscillator via the SET. As long as the amplitude of the oscillations around its equilibrium position is small compared to the distance $d$ separating the oscillator and the SET island, the gate capacitance $C_{g}(x)$ can be treated as linear in $x$. As a consequence, we obtain position-dependent dimensionless tunneling rates of the form

$$
\begin{aligned}
& \Gamma_{L}^{+(-)}(x)=(-)\left[\Delta_{L}-\kappa \frac{x}{x_{0}}\right] f\left((-)\left[\Delta_{L}-\kappa \frac{x}{x_{0}}\right] e V_{d s}\right) \\
& \Gamma_{R}^{+(-)}(x)=(-)\left[\Delta_{R}+\kappa \frac{x}{x_{0}}\right] f\left((-)\left[\Delta_{R}+\kappa \frac{x}{x_{0}}\right] e V_{d s}\right),
\end{aligned}
$$

where the coefficients ${ }^{29}$

$$
\begin{aligned}
& \Delta_{L}=\frac{1}{2}+\left(2 N-2 N_{g}+1\right) \frac{E_{c}}{e V_{d s}}-\kappa N, \\
& \Delta_{R}=\frac{1}{2}-\left(2 N-2 N_{g}+1\right) \frac{E_{c}}{e V_{d s}}+\kappa N
\end{aligned}
$$

are the position-independent part of the full dimensionless rate $\Gamma_{i}^{\sigma}(x)$, which fulfill $\Delta_{L}+\Delta_{R}=1$, and

$$
\kappa=m \omega_{0}^{2} x_{0}^{2} /\left(e V_{d s}\right)
$$

is a dimensionless coupling parameter that will play an important role in the following. Note that $\Delta_{L}$ and $\Delta_{R}$ can become negative in the strong-coupling limit. The average dimensionless current in the presence of position-dependent rates can be calculated as an average of the different rates weighted by the probability to find the oscillator at a position $x$ : 


$$
\begin{aligned}
I & =\int_{-\infty}^{\infty} d x\left[P_{N+1}(x) \Gamma_{L}^{+}(x)-P_{N}(x) \Gamma_{L}^{-}(x)\right] \\
& =\int_{-\infty}^{\infty} d x\left[P_{N}(x) \Gamma_{R}^{+}(x)-P_{N+1}(x) \Gamma_{R}^{-}(x)\right],
\end{aligned}
$$

with $P_{N(N+1)}(x)$ the probability to find the oscillator at position $x$ while the island charge state is $N(N+1)$.

In the zero-temperature limit, the Fermi functions in Eqs. (5) are in fact Heaviside step functions that determine the possible transport direction as a function of the position of the oscillator. Indeed, at zero temperature, $x^{L}=\Delta_{L} x_{0} / \kappa$ and $x^{R}=-\Delta_{R} x_{0} / \kappa$ define points where the current direction at leads $L$ and $R$ changes sign. For $x^{R}<x$ current in the right junction can only be directed towards the island while in the opposite case only charge transfer from the island to the right lead is possible. Equivalently, transfer through the left junction is allowed from the island to the lead if $x<x^{L}$ and from the lead to the island otherwise. It is interesting to note that transport can be blocked altogether via this mechanism. For example, if $N+1$ electrons are on the island and the oscillator is in position $x>x^{L}$, transport of the extra charge from the island to any lead is effectively forbidden, our choice of bias direction imposing $x^{R}<x^{L}$.

The canonical way of dealing with a SET in the sequential tunneling regime is to introduce a master equation for the different charge states of the island. If the oscillator is coupled to a nanomechanical oscillator, such a simple master equation cannot be written, since the tunneling rates depend on the stochastic evolution of the oscillator. Following Ref. 14 we can introduce the probability distributions $P_{N}(x, u ; t)$ and $P_{N+1}(x, u ; t)$ to find, at a time $t$, the oscillator at position $x, u$ in phase space and the SET in charge state $N$ and $N+1$, respectively, and derive a master equation for these new objects:

$$
\begin{aligned}
\frac{\partial}{\partial t} P_{N}(x, u ; t)= & \omega_{0}^{2} x \frac{\partial}{\partial u} P_{N}(x, u ; t)-u \frac{\partial}{\partial x} P_{N}(x, u ; t) \\
& +\left[\Gamma_{L}^{+}(x)+\Gamma_{R}^{-}(x)\right] P_{N+1}(x, u ; t) \\
- & {\left[\Gamma_{R}^{+}(x)+\Gamma_{L}^{-}(x)\right] P_{N}(x, u ; t), } \\
\frac{\partial}{\partial t} P_{N+1}(x, u ; t)= & \omega_{0}^{2}\left(x-x_{0}\right) \frac{\partial}{\partial u} P_{N+1}(x, u ; t)-u \frac{\partial}{\partial x} P_{N+1}(x, u ; t) \\
& -\left[\Gamma_{L}^{+}(x)+\Gamma_{R}^{-}(x)\right] P_{N+1}(x, u ; t) \\
& +\left[\Gamma_{R}^{+}(x)+\Gamma_{L}^{-}(x)\right] P_{N}(x, u ; t) .
\end{aligned}
$$

As pointed out in Ref. 14, when the coupling between the oscillator and the SET is weak $(\kappa \ll 1)$ and when the gate voltage $V_{g}$ is such that the system is tuned far from the Coulomb-blockade region, one can make the approximation that $x^{L} \rightarrow \infty$ and $x^{R} \rightarrow-\infty$ and then write the tunneling rates as

$$
\Gamma_{L}^{+}(x)=\Delta_{L}-\kappa \frac{x}{x_{0}}, \quad \Gamma_{L}^{-}(x)=0
$$

$$
\Gamma_{R}^{+}(x)=\Delta_{R}+\kappa \frac{x}{x_{0}}, \quad \Gamma_{R}^{-}(x)=0 .
$$

This weak-coupling form of Eq. (5) effectively corresponds to neglecting any back currents and the possibility of position-induced current blockade. However, the master equation is then simple enough to allow analytical solutions.

In this work, we will not study the effect of extrinsic damping (i.e., a finite quality factor of the oscillator) and of finite temperatures, since they were discussed extensively in Refs. 14 and 15.

\section{DYNAMICS OF THE OSCILLATOR IN THE STRONG-COUPLING REGIME}

In the weak-coupling limit $\kappa \ll 1$, it was found ${ }^{14}$ that the interaction between the SET and the oscillator introduces an intrinsic damping mechanism. The damping, characterized by a decay rate $\gamma=\kappa \epsilon^{2}$ (in units of $\tau_{t}^{-1}$ ), leads to a steadystate solution for the probability distributions $P_{N(N+1)}(x, u)$. In particular, the probability distributions $P_{N(N+1)}(x)$ $=\int d u P_{N(N+1)}(x, u)$, from which one can calculate the average current, have been shown to be well approximated by Gaussians centered at $x=0$ and $x=x_{0}$ for $P_{N}$ and $P_{N+1}$, respectively.

One of the main goals of this work is to study deviations from the weak-coupling behavior. Without the simplifications possible for $\kappa \ll 1$ leading to Eq. (10), the stationary probability distributions $P_{N(N+1)}(x, u)$ can no longer be investigated analytically and numerical methods must be used. In this work, we used a Monte Carlo approach to simulate the stochastic nature of the SET-nanomechanical-oscillator system in the parameter regime where the typical mechanical energy $m \omega_{0}^{2} x_{0}^{2}$ is comparable to the bias energy $e V_{d s}$. Details of our implementation of the Monte Carlo method are given in the Appendix.

We study the probability distribution of the oscillator in the charge-degenerate case, where $\left\langle P_{N}\right\rangle=\left\langle P_{N+1}\right\rangle$, where $\left\langle P_{N}\right\rangle=\int d x P_{N}(x)$. At this point the current flowing through the SET is maximal. In the presence of the oscillator, charge degeneracy is reached when $\Delta_{L}=1 / 2+\kappa / 2$. This relation, exact in the weak-coupling limit, has been empirically verified over the whole range of $\kappa$ studied. In the weak-coupling limit, this relation can be shown using $\left\langle P_{N+1}\right\rangle=\Delta_{R}$ familiar from two-state SET's. In our case, at the degeneracy point symmetry considerations impose $\langle x\rangle=x_{0} / 2$ and the position dependence of the rate $\Gamma_{R}^{+}(x)$ must be taken into account, such that $\left\langle P_{N+1}\right\rangle=\frac{1}{2}=\Delta_{R}+\kappa\left\langle x / x_{0}\right\rangle$. This effectively corresponds to $\left\langle P_{N+1}\right\rangle=\Delta_{L}-\kappa / 2$.

As can be seen from Fig. 2, as $\kappa$ is increased, the stationary position probability distribution evolves continuously from the weak-coupling Gaussian form to a distribution showing two sharp peaks at $x=0$ and $x=x_{0}$ in the limit where $\kappa=1$. This evolution is the result of a sharpening of each of the two subdistributions $P_{N(N+1)}(x)$ around their equilibrium position when $\kappa$ is increased, allowing one to resolve the two subdistributions individually. This should not only be seen as a natural consequence of the fact that the typical distance $x_{0}$ 

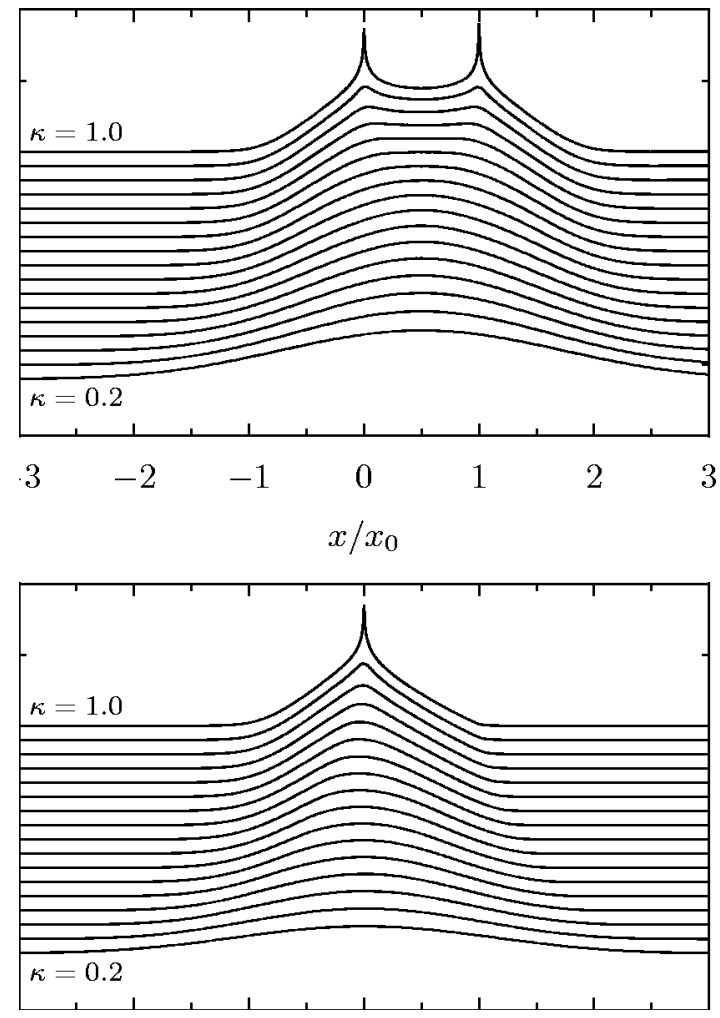

FIG. 2. Upper panel: total probability distribution $P(x)=P_{N}(x)$ $+P_{N+1}(x)$ of the oscillator's position for different values of the coupling constant $\kappa$ [defined in Eq. (7)]. Lower panel: probability distribution $P_{N}(x)$ to find the oscillator at position $x$ if the SET is in charge state $N . P_{N+1}(x)$ can be obtained by the symmetry relation $P_{N+1}(x)=P_{N}(1 / 2-x)$. In both panels, lines are shifted for clarity by $2 \kappa$ and the difference between neighboring curves is $\Delta \kappa=0.05$. All calculations were done at $\epsilon=0.3$ and at the charge-degeneracy point. For the definition of $\epsilon$, see Eq. (4).

scales like $\sqrt{\kappa}$. In fact, the main cause of the appearance of the two sharp peaks is that small-amplitude oscillations about each of the two equilibrium points become very stable when $\kappa$ is increased.

We also note that the qualitative shape of each subdistribution evolves when $\kappa$ is increased. While at low coupling the subdistribution $P_{N}(x)\left[P_{N+1}(x)\right]$ is symmetric about $x=0$ $\left[x=x_{0}\right]$, this is not the case for $\kappa \geqslant 0.4$. This asymmetry arises only at higher coupling since, for low $\kappa$, the probability to find the oscillator at $x<x^{R}$ or $x>x^{L}$ is negligible. When $\kappa$ $\gtrsim 0.4$, the probability of the oscillator being in a region transport is forbidden becomes important. Symmetry breaking arises since these regions are located only on one side of each equilibrium point.

Finally, we note that the important changes in $P_{N(N+1)}(x)$ which accompany a variation in $\kappa$ are also seen in the stationary velocity subdistributions $P_{N(N+1)}(u)=\int d u P_{N(N+1)}$ $\times(x, u)$, which approximatively follow $P_{N}\left(u / \epsilon u_{0}\right)$ $\simeq P_{N}\left(x / x_{0}\right)$ and $P_{N+1}\left(u / \epsilon u_{0}\right) \simeq P_{N+1}\left(\left(x-x_{0}\right) / x_{0}\right)$, where $u_{0}$ $=x_{0} / \tau_{t}$ is the typical velocity scale in the problem. This can be seen in the two-dimensional phase-space distributions shown in Fig. 3, where both $P(u)$ and $P(x)$ are shown to become more peaked when $\kappa$ is increased.
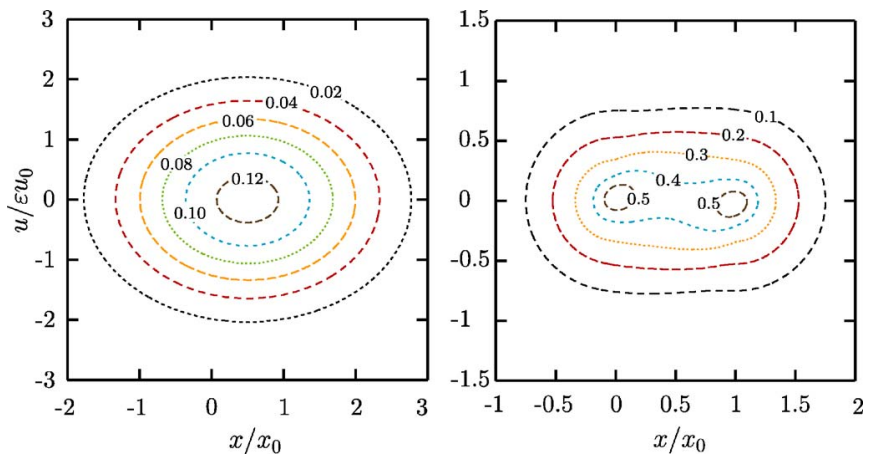

FIG. 3. (Color online) Two-dimensional phase-space distributions $P(x, u)=P_{N}(x, u)+P_{N+1}(x, u)$, for $\kappa=0.2$ (left panel) and $\kappa$ $=0.9$ (right panel).

For $\kappa>1$, our numerical investigations show that the current is strongly suppressed, rendering the intrinsic damping mechanism discussed at the beginning of this section ineffective. In this case, the system cannot be characterized by a steady-state probability distribution and our model is not appropriate. Therefore, we only studied the parameter range $\kappa \leqslant 1$.

A similar reasoning applies to the Coulomb-blockade region, where the damping of the oscillator's motion is severely suppressed. However, numerically finding a steadystate solution close to the degeneracy point is possible.

\section{AVERAGE CURRENT}

The average current flowing through the SET is closely tied to the oscillator's position distributions via the positiondependent tunneling rates. Consequently, one expects that the deviations from the weak-coupling behavior observed in $P(x)=P_{N}(x)+P_{N+1}(x)$ would affect the current characteristics when $\kappa$ is increased.

Just like in the previous section, we focus on the degeneracy point where the average charge state of the island is $N+1 / 2$. At this point, the weak-coupling theory predicts ${ }^{14}$ that the current decreases linearly with increasing $\kappa: I$ $=\frac{1-\kappa}{4}\left(e / \tau_{t}\right)$. This decrease in the current can be explained in a qualitative way by the reduction of the overlap of the distributions $P_{N}(x)$ and $P_{N+1}(x)$ as the coupling is increased, each distribution becoming more localized around its equilibrium point; see Fig. 2.

Figure 4 shows the average current as a function of $\kappa$. Like in the weak-coupling limit, the localization of each probability distribution around its equilibrium point leads to an overall decrease in the current when the coupling grows stronger. For $\kappa \gtrsim 0.3$, however, we see that the numerical results deviate from the weak-coupling behavior: for stronger coupling the current is higher than the weak-coupling result. This can be explained using the rates given by Eq. (10). When either $\int_{x^{L}}^{\infty} d x P_{N+1}$ or $\int_{-\infty}^{x^{R}} d x P_{N}$ is not negligible, these rates allow unphysical backward currents that are not present in the full master equation. For example, a point located at $x>x^{L}$ in the steady-state probability distribution $P_{N+1}(x, u)$ would contribute negatively to the average current when us- 


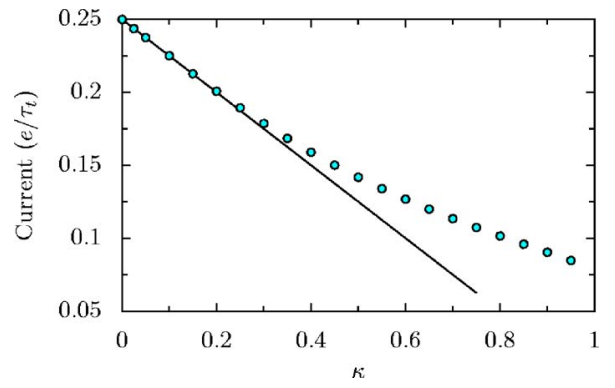

FIG. 4. (Color online) Current (in units of $e / \tau_{t}$ ) as a function of $\kappa$ at the degeneracy point $\left\langle P_{N}\right\rangle=\left\langle P_{N+1}\right\rangle$, for $\epsilon=0.3$. The dots are the results of the Monte Carlo calculation, and the solid line is the analytic form found within the weak-coupling approximation.

ing the rates calculated within the weak-coupling approximation while it would not contribute to the current when taking into account the full expression for the rates given in Eq. (6).

Over the range of frequencies that we studied numerically $(0.1 \leqslant \epsilon \leqslant 0.4)$, the current was found to be practically independent of $\epsilon$ for all but the strongest couplings ( $\kappa \gtrsim 0.8$ ). For instance, at $\kappa=0.9$, the difference between the calculated currents at $\epsilon=0.1$ and $\epsilon=0.4$ is of the order of a few percent.

\section{NOISE AND HIGHER CUMULANTS IN THE STATIC REGIME}

Originally, interest in SET's was motivated by suppression of the current in the Coulomb-blockade regime and the high sensitivity of the current to small variations of the gate voltage. However, it is clear that a complete description of the transport processes in these devices requires not only knowledge of the current, but also of current-current correlations like, e.g., the current noise. ${ }^{30,31}$ Recently, higher-order correlations have also been studied both theoretically and experimentally in nanoscale devices, in the framework of full counting statistics (FCS) (see Ref. 32 for a collection of articles on this topic and Refs. 33 and 34 for a description of FCS in the context of transport through SET's). The FCS approach consists in studying the probability distribution $\mathcal{P}_{n}\left(t_{0}\right)$ that $n$ electrons are transferred through one lead of the SET within a time period $t_{0}$, in the limit where $t_{0}$ is by far the longest time scale in the problem. The full information about the transport properties is contained in the cumulants of this distribution function, the first three of which are given by the average $\mu_{1}=\langle n\rangle$, the variance $\mu_{2}=\left\langle n^{2}\right\rangle-\langle n\rangle^{2}$, and the skewness $\mu_{3}=\left\langle(n-\langle n\rangle)^{3}\right\rangle$, which measures the asymmetry of the distribution. For example, the current $I=e\langle n\rangle / t_{0}$ is proportional to the mean of this distribution, while the zerofrequency shot-noise power $S(0)=2 e^{2} \mu_{2} / t_{0}$ is determined by its second cumulant.

In this section, we study in detail the second and third cumulants of the probability distribution function $\mathcal{P}_{n}\left(t_{0}\right)$ in the case of a coupled SET-nanomechanical system.

\section{A. Weak-coupling case}

It is instructive to start by considering the weak-coupling case $\kappa \ll 1$, since in this regime we can calculate the noise and higher cumulants without resorting to Monte Carlo simulations by solving directly for $\left\langle n^{i}\left(t_{0}\right)\right\rangle$ in the long-time limit $\left(t_{0} \gamma \gg 1\right)$. In this section, we generalize the work that was done in Ref. 15 where a method to calculate the current noise using the moments of the steady-state probability distribution $P_{N(N+1)}(x, u)$ of the oscillator in phase space was described. In this approach, the current noise is calculated from the solution of the equation of motion of $\left\langle n^{2}(t)\right\rangle$, the average square of the number of charges that went through a lead in a time $t$. Here, we extend this method for the calculation of higher cumulants by deriving the equation of motion for the general quantity $\left\langle n^{m}(t)\right\rangle$ from which the $m$ th cumulant can be extracted.

To proceed, we write a master equation for the probability $P_{N(N+1)}^{n}(x, u ; t)$ to find, at time $t$, the oscillator at position $x$ with velocity $u$, the island being in charge state $N(N+1)$, with $n$ charges having passed through a lead of the SET in the interval $[0 ; t]$. We will again make the assumptions leading to Eq. (10). Considering for definitiveness the left lead, at zero temperature and neglecting any extrinsic damping, one finds 15

$$
\begin{aligned}
\frac{\partial}{\partial t} P_{N}^{n}(x, u ; t)= & \omega_{0}^{2} x \frac{\partial}{\partial u} P_{N}^{n}(x, u ; t)-u \frac{\partial}{\partial x} P_{N}^{n}(x, u ; t) \\
+ & \Gamma_{L}^{+}(x) P_{N+1}^{n-1}(x, u ; t)-\Gamma_{R}^{+}(x) P_{N}^{n}(x, u ; t), \\
\frac{\partial}{\partial t} P_{N+1}^{n}(x, u ; t)= & \omega_{0}^{2}\left(x-x_{0}\right) \frac{\partial}{\partial u} P_{N+1}^{n}(x, u ; t)-u \frac{\partial}{\partial x} P_{N+1}^{n}(x, u ; t) \\
& -\Gamma_{L}^{+}(x) P_{N+1}^{n}(x, u ; t)+\Gamma_{R}^{+}(x) P_{N}^{n}(x, u ; t),
\end{aligned}
$$

where the rates are taken from Eq. (10). Defining the coupled moments $\left\langle x^{j} u^{k} n^{m}\right\rangle$ and $\left\langle x^{j} u^{k} n^{m}\right\rangle_{N+1}$ as

$$
\begin{gathered}
\left\langle n^{m} x^{j} u^{k}\right\rangle=\sum_{n} n^{m} \int d u \int d x x^{j} u^{k}\left[P_{N}^{n}(x, u ; \tau)+P_{N+1}^{n}(x, u ; \tau)\right] \\
\left\langle n^{m} x^{j} u^{k}\right\rangle_{N+1}=\sum_{n} n^{m} \int d u \int d x x^{j} u^{k} P_{N+1}^{n}(x, u ; \tau),
\end{gathered}
$$

one can calculate the equation of motion for these quantities using Eq. (11). With $x$ in units of $x_{0}$ and $u$ in units of $u_{0}$, one finds

$$
\begin{aligned}
\frac{d}{d \tau}\left\langle x^{j} u^{k} n^{m}\right\rangle_{N+1}= & -k \epsilon^{2}\left[\left\langle x^{j+1} u^{k-1} n^{m}\right\rangle_{N+1}-\left\langle x^{j} u^{k-1} n^{m}\right\rangle_{N+1}\right] \\
& +j\left\langle x^{j-1} u^{k+1} n^{m}\right\rangle_{N+1}-\left\langle x^{j} u^{k} n^{m}\right\rangle_{N+1} \\
& +\Delta_{R}\left\langle x^{j} u^{k} n^{m}\right\rangle+\kappa\left\langle x^{j+1} u^{k} n^{m}\right\rangle
\end{aligned}
$$




$$
\begin{aligned}
\frac{d}{d \tau}\left\langle x^{j} u^{k} n^{m}\right\rangle= & -k \epsilon^{2}\left[\left\langle x^{j+1} u^{k-1} n^{m}\right\rangle-\left\langle x^{j} u^{k-1} n^{m}\right\rangle_{N+1}\right] \\
& +j\left\langle x^{j-1} u^{k+1} n^{m}\right\rangle+\sum_{i=0}^{m-1}\left(\begin{array}{c}
m \\
i
\end{array}\right)\left[\Delta_{L}\left\langle x^{j} u^{k} n^{i}\right\rangle_{N+1}\right. \\
& \left.-\kappa\left\langle x^{j+1} u^{k} n^{i}\right\rangle_{N+1}\right] .
\end{aligned}
$$

Here, averages that are independent of $n$ (averages of the form $\left\langle x^{j} u^{k} n^{0}\right\rangle$ ) are time independent and can be evaluated in the stationary limit; i.e., Eqs. (13a) and (13b) can be used to generate a closed linear system of equations. ${ }^{35}$ The terms $\left\langle x^{j} u^{k}\right\rangle$ of order $j+k=c$ are connected to the terms $\left\langle x^{j} u^{k}\right\rangle_{N+1}$ of order $j+k=c-1$. This means that to solve for a moment $\left\langle x^{j} u^{k}\right\rangle$, we must use the $c+1$ equations of the type of Eq. (13b) where $j+k=c$ and the $c$ equations of the type of Eq. (13a) where $j+k=c-1$. This method can be used to calculate any moment of the form $\left\langle x^{j} u^{k}\right\rangle$ and $\left\langle x^{j} u^{k}\right\rangle_{N+1}$. Knowledge of $\left\langle x^{j} u^{k}\right\rangle$ enables one to calculate the long-time behavior of the coupled moments of the charge and oscillator's position in phase space $\left\langle x^{j^{\prime}} u^{k^{\prime}} n^{i}\right\rangle$ and, thus, the $i$ th moment $\left\langle n^{i}\right\rangle$ of $\mathcal{P}_{n}$.

The ratio of the zero-frequency shot-noise power and the average current (times $2 e$ ) or, equivalently, the ratio of the second and first cumulants of $\mathcal{P}_{n}$ is called the Fano factor and is readily calculated using this approach. Since it shows a complex dependence on the coefficients $\Delta_{L}$ and $\Delta_{R}$ and on the parameters $\kappa$ and $\epsilon$, it is convenient to expand the result in powers of $\kappa$. Introducing a parameter $\alpha$ defined via $\alpha$ $=\Delta_{L}-(1+\kappa) / 2$ [or, equivalently, $\alpha=-\Delta_{R}+(1-\kappa) / 2$ ], which measures the difference between $\Delta_{L}$ and its value at the degeneracy point, one can write down the Fano factor in a way that underlines its symmetry with respect to this point:

$$
\begin{aligned}
\frac{S(0)}{2 e I}= & \frac{1}{2}+2 \alpha^{2}+4 \alpha^{2} \kappa+6 \alpha^{2} \kappa^{2}+\left(\frac{1}{2}-2 \alpha^{2}\right) \frac{\kappa}{\epsilon^{2}} \\
& -\left(\frac{1}{2}+2 \alpha^{2}\right) \frac{\kappa^{2}}{\epsilon^{2}}+O\left(\kappa^{3}\right) .
\end{aligned}
$$

For $\epsilon \ll 1$, the Fano factor is dominated by the term $\sim \kappa / \epsilon^{2}$, like in the case where one considers a system of two SET's coupled by an oscillator. ${ }^{36}$ Finally, we note that current conservation implies that the Fano factor is identical in both leads. ${ }^{37}$

Equation (13) is one of the main results of our article, as it allows for the calculation of higher cumulants of the current by integrating the equation of motion for the moments of the form $\left\langle x^{j} u^{k} n^{m}(t)\right\rangle$ with $m>0$. For example, we calculated the normalized third cumulant $\mu_{3} /\langle n\rangle$ of $\mathcal{P}_{n}\left(t_{0}\right)$. The results are presented in Fig. 5. We stress that these results have been obtained by integrating the equation motion for $\left\langle n^{3}(t)\right\rangle$ valid in the weak-coupling regime and not via a Monte Carlo simulation. Starting from the value $1 / 4$ at $\kappa$ $=0,{ }^{33}$ the normalized third cumulant decreases rapidly when $\kappa$ is increased. On further increase of $\kappa$, it reaches a minimum at an $\epsilon$-dependent value of $\kappa$. The inset of Fig. 5 shows that the leading contribution to the normalized third cumulant in the weak-coupling regime is of the form $\epsilon^{-4}$. As a consequence, we note that the asymmetry of the probability

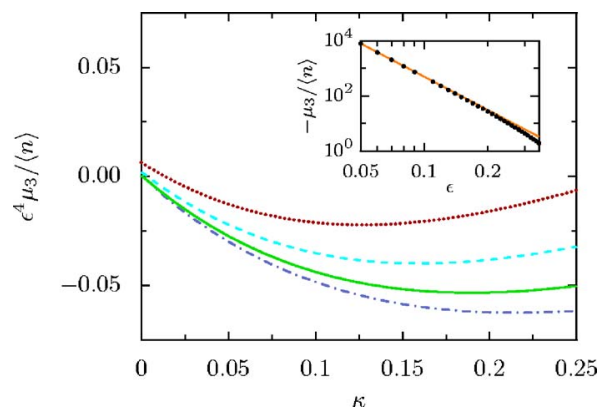

FIG. 5. (Color online) Normalized third cumulant as a function of $\kappa$ for different values of $\epsilon$, as calculated within the weakcoupling approximation and scaled by $\epsilon^{4}$. Dotted line: $\epsilon=0.4$. Dashed line: $\epsilon=0.3$. Solid line: $\epsilon=0.2$. Dash-dotted line: $\epsilon=0.1$. The inset shows the $\epsilon$ dependence of the normalized third cumulant at $\kappa=0.1$ (symbols), as calculated within the weak-coupling approximation. The solid line is a fit to a power law $\sim \epsilon^{-4}$. These results were obtained by integrating the equation of motion for $\left\langle n^{3}(t)\right\rangle$ following from Eq. (13).

distribution $\mathcal{P}_{n}$, which is determined by $\mu_{3}$, can effectively be tuned by changing the frequency of the oscillator or $\tau_{t}$. The scaled quantity $\epsilon^{4} \mu_{3} /\langle n\rangle$ shows contributions of higherorder corrections in $\epsilon$ to the normalized third cumulant.

\section{B. Higher coupling}

It is unfortunately not straightforward to generalize the previously described method for calculating the cumulants of $\mathcal{P}_{n}$ outside the weak-coupling regime. The presence of $x$-dependent Fermi functions in the tunneling rates as well as the possibility of charge flow against the direction set by the bias voltage due to the position of the oscillator gives rise to a system of equations that is not closed and cannot be solved analytically. Even if we neglect transport against the dominant direction of the current $\Gamma_{j}^{-}(x) \simeq 0$, but keep the position dependence of the Fermi distributions in $\Gamma_{j}^{+}(x)$, it is still not possible to derive a system of equations coupling only objects of the form $\left\langle x^{j} u^{k} n^{m}\right\rangle$. Therefore, we will use a numerical approach to evaluate the cumulants of $\mathcal{P}_{n}$.

Indeed, the Monte Carlo method described in the Appendix can be used to measure the FCS of electron transport in the same way as it can be done in experiments. ${ }^{38} \mathrm{~A}$ very long Monte Carlo run is divided into intervals of duration $t_{0}$ $\gg \gamma^{-1}$; here, $\gamma=\kappa \epsilon^{2}$ is the damping constant defined at the beginning of Sec. III and $\gamma^{-1}$ is the longest intrinsic time scale of the problem. By counting the number of charges going through one lead during each interval, one can reconstruct the probability distribution $\mathcal{P}_{n}\left(t_{0}\right)$ and from it calculate the cumulants.

We study current correlations at the charge degeneracy point, where the average charge state of the island is $N$ $+1 / 2$. The top panel of Fig. 6 compares the weak-coupling Fano factor to the numerical Monte Carlo results for different values of the coupling parameter $\kappa$. Naturally, for $\kappa$ $\lessgtr 0.2$, the agreement between the numerical results and those obtained analytically is very good. Beyond this point, the numerically calculated Fano factor shows an interesting non- 

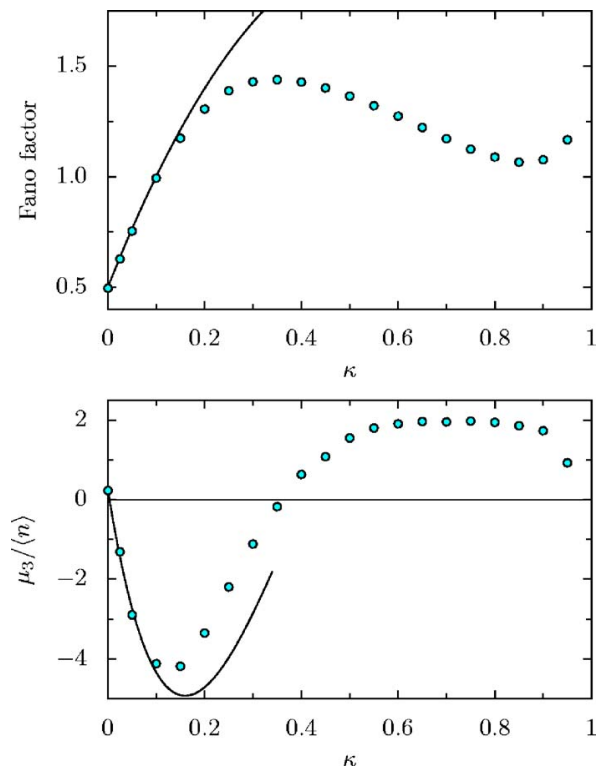

FIG. 6. (Color online) Upper panel: Fano factor as a function of $\kappa$ at the degeneracy point $\left\langle P_{N}\right\rangle=\left\langle P_{N+1}\right\rangle$. The dots are the results of the numerical calculation, and the solid line is the analytic form found within the weak-coupling approximation. Lower panel: normalized third cumulant $\mu_{3}$ of the probability distribution $\mathcal{P}_{n}$. For both panels, $\epsilon=0.3$.

monotonic behavior, with a maximum at $\kappa \sim 0.35$ and a minimum at $\kappa \sim 0.85$. The lower panel of Fig. 6 also shows the evolution of the normalized third cumulant of $\mathcal{P}_{n}$, giving the asymmetry of this probability distribution about its mean $\langle n\rangle$. Starting from the $\kappa=0$ value of $1 / 4$ derived for a simple SET device, our results show that this quantity is, in the weak-coupling limit, very sensitive to variations of $\kappa$. Indeed, the normalized third cumulant changes sign twice in the region $\kappa \leqq 0.35$, reaching a maximum value approximatively in the middle of this region. This contrasts with the strong-coupling behavior: $\mu_{3} /\langle n\rangle$ stays practically constant for $0.5 \lesssim \kappa \lesssim 0.9$.

We will now address the question how the previous results are modified when changing the frequency of the oscillator. Figure 7 shows the dependence of the Fano factor and the normalized third cumulant as a function of $\kappa$ for different values of $\epsilon$. First, we note that the actual value of the Fano factor is increased dramatically for lower oscillator frequencies, as expected from the term $\sim \kappa / \epsilon^{2}$, which dominates in the low-frequency regime. In the weak-coupling region $(\kappa$ $\lesssim 0.3$ ), the magnitude of the normalized third cumulant is also heavily affected by a change in frequency, in agreement with the weak-coupling leading-order dependence $\sim \epsilon^{-4}$. Despite these major changes in magnitude of both the Fano factor and the normalized third cumulant, the overall qualitative effect of an increase in coupling does not seem to depend heavily on $\epsilon$ in the frequency range we investigated. In particular, the position of the maximum in the Fano factor remains constant. Also, the normalized third cumulant always shows a change of sign, albeit at an $\epsilon$-dependent value of $\kappa$, and goes to a positive for $\kappa \rightarrow 1$. Remarkably, the value of the normalized third cumulant is much less sensitive to $\epsilon$
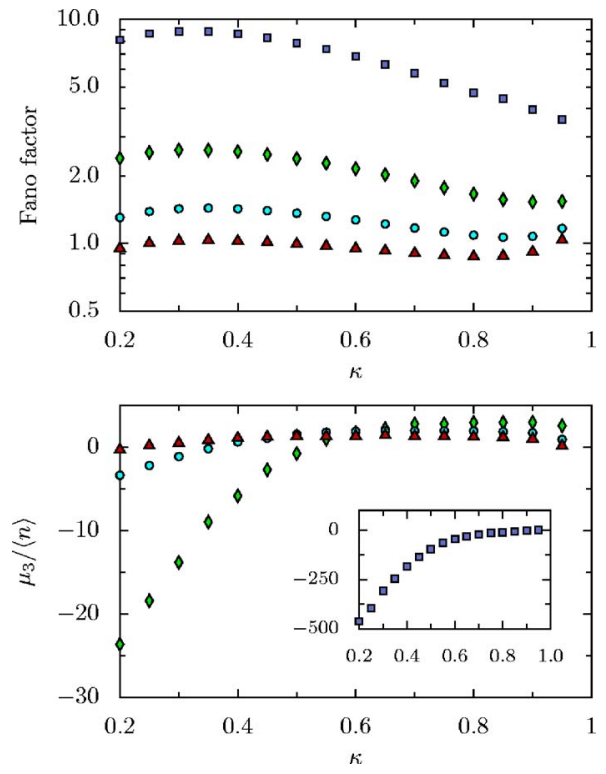

FIG. 7. (Color online) Fano factor (upper panel) and normalized third cumulant (lower panel and inset) as a function of $\kappa$ for different values of $\epsilon$ : $\epsilon=0.1$ (squares in the upper panel and in the inset), $\epsilon=0.2$ (diamonds), $\epsilon=0.3$ (circles), and $\epsilon=0.4$ (triangles). Note the logarithmic $y$ axis in the upper panel.

in the strong-coupling regime. This might be the signature of a transport regime in the $\kappa \rightarrow 1$ region that is radically different of the one found for $\kappa \simeq 0.2$.

\section{FREQUENCY-DEPENDENT NOISE}

In systems that exhibit internal dynamics like the one we study, it is especially interesting to look at the frequency dependence of the current-current correlations. In Ref. 15, the frequency-dependent noise $S(\omega)$ of a SET weakly coupled to a nanomechanical oscillator was thoroughly studied. It was found that the noise spectrum shows only two peaks at finite frequency at $\omega_{0}^{\prime}$ and $2 \omega_{0}^{\prime}$, where $\omega_{0}^{\prime}$ $=\omega_{0} \sqrt{1-\kappa}$ is the effective frequency of the damped harmonic oscillator. In this section, we extend the work of Ref. 15 by studying the frequency-dependent noise power $S(\omega)$ in the strong-coupling regime $(0.2 \leqslant \kappa<1)$.

In order to calculate the frequency-dependent noise using our Monte Carlo method, we follow the approach developed by MacDonald, ${ }^{31,39}$ which was used recently to study the noise properties of mesoscopic systems, including coupled SET-nanomechanical systems in the weak-coupling regime. In general, the current-noise power at junction $a$ is defined as the Fourier transform of the current autocorrelation function $K_{i, i}$ at junction $i$,

$$
S_{i, i}(\omega)=2 \int_{-\infty}^{\infty} d \tau \cos (\omega \tau) K_{i, i}(\tau),
$$

where

$$
K_{i, i}(\tau)=\left\langle I_{i}(t+\tau) I_{i}(t)\right\rangle-\left\langle I_{i}\right\rangle^{2} .
$$

To proceed with the MacDonald approach, $I_{i}$ and, therefore, $I_{i}-\left\langle I_{i}\right\rangle$ must be assumed to be statistically fluctuating vari- 


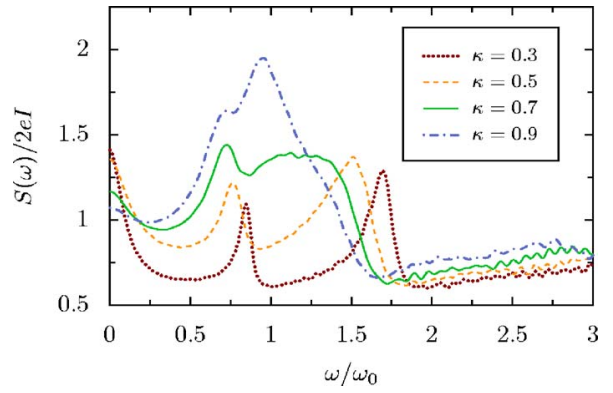

FIG. 8. (Color online) Frequency-dependent noise power beyond the weak-coupling approximation. For each curve, the SET is tuned to the charge-degeneracy point and $\epsilon=0.3$.

ables, such that the autocorrelation function $K_{i, i}$ is independent of $t$. In this case, the MacDonald formula relates the fluctuation $\delta n$ about the average of the number of charges $n$ that went through a junction in time $\tau$,

$$
\delta n_{i}(\tau)=n_{i}(\tau)-\left\langle I_{i}\right\rangle \tau=\int_{t}^{t+\tau} d t^{\prime}\left[I_{i}\left(t^{\prime}\right)-\left\langle I_{i}\right\rangle\right]
$$

to the current-noise power via

$$
S_{i, i}(\omega)=2 \omega \int_{0}^{\infty} d \tau \sin (\omega \tau) \frac{\partial}{\partial \tau}\left\langle\left[\delta n_{i}(\tau)\right]^{2}\right\rangle,
$$

where $\left\langle\left[\delta n_{i}(\tau)\right]^{2}\right\rangle=\left\langle n_{i}^{2}(\tau)\right\rangle-\left\langle I_{i}\right\rangle^{2} \tau^{2}$. Since $\left\langle n^{2}(\tau)\right\rangle$ and $\left\langle I_{i}\right\rangle$ are easily accessible through the Monte Carlo simulation, $S(\omega)$ can be calculated by taking a numerical time derivative of $\left\langle\left[\delta n_{i}(\tau)\right]^{2}\right\rangle$ and then evaluating the Fourier sine transform. Note that we consider only the particle current fluctuations here. The electrical current noise at finite frequencies includes a contribution from displacement currents, which depend on the capacitive couplings between the island and the leads. ${ }^{37}$ Since we assume that our frequencies of interest are much smaller than the relevant $R C$ frequencies, we can neglect the displacement currents here; see, e.g., the discussion in Refs. 40 and 41.

The results of the Monte Carlo simulation are shown in Fig. 8. Like in the weak-coupling case, $S(\omega)$ shows two main finite-frequency features. Surprisingly, even for strong coupling, we do not find any features for frequencies higher than $2 \omega_{0}$. We find a low-frequency peak that defines the frequency $\omega_{0}^{\prime}$ (which will be different from the weak-coupling prediction $\omega_{0} \sqrt{1-\kappa}$ in the general case). The second feature evolves from the peak located at $2 \omega_{0}^{\prime}$ predicted by the weakcoupling theory. While both peaks are considerably broadened by an increase in the coupling strength, their respective shapes evolve in a qualitatively different way. Whereas the first peak is shifted in absolute frequency, the second peak broadens in a very asymmetric way, with much of the weight shifting to lower frequencies. The slope of its left shoulder decreases with increasing $\kappa$ until it forms a plateau at around $\kappa \sim 0.7$. On increasing $\kappa$ even further, the two peaks merge, leading to super-Poissonian frequency-dependent noise throughout the frequency range $\omega<1.5 \omega_{0}$.

Figure 9 shows the position of the maxima of the first peak in the frequency-dependent noise power as a function

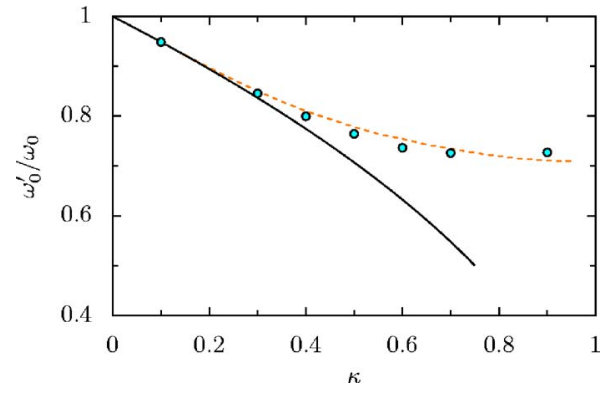

FIG. 9. (Color online) Position $\omega_{0}^{\prime}$ of the first peak in the frequency-dependent noise power as a function of $\kappa$. The solid line gives the weak-coupling prediction $\omega_{0} \sqrt{1-\kappa}$, the data points are the numerical Monte Carlo results, and the dashed line was obtained using an effective damping constant; see text.

of $\kappa$. By comparing the position of the first peak extracted from the curves shown in Fig. 8 (data points in Fig. 9) to the weak-coupling prediction $\omega_{0}^{\prime}=\omega_{0} \sqrt{1-\kappa}$ (solid line in Fig. 9), we find quantitative agreement only for $\kappa \lesssim 0.2$. Beyond this point, the ratio $\omega_{0}^{\prime} / \omega_{0}$ still decreases, albeit slowly, when $\kappa$ is increased. It reaches a saturation value $\omega_{0}^{\prime} \sim 0.7 \omega_{0}$ for $\kappa$ $\geq 0.7$.

This behavior can be understood by interpreting the frequency shift in terms of an effective damping mechanism caused by electron tunneling. Since there is no damping without current, the natural modification of the weakcoupling damping constant $\gamma=\kappa \epsilon^{2}$ in the strong-coupling regime is to renormalize the weak-coupling result by the probability $P^{*}$ to find the oscillator in a position where in principle current is allowed-i.e., for $x^{R}<x$ and charge state $N$ or $x<x^{L}$ and charge state $N+1$. Defining a renormalized damping constant $\gamma^{s c}=P^{*} \kappa \epsilon^{2}$, it is possible to estimate the position of the first peak as a function of $\kappa$ using values of $P^{*}$ extracted from curves presented in Fig. 2. The result is shown as the dashed curve in Fig. 9 and agrees with the data points obtained by the Monte Carlo method in a quantitative way.

\section{CONCLUSION}

In this paper, we have studied the strong-coupling limit of a SET coupled to a classical harmonic oscillator. We have used a combination of a master-equation approach and a numerical Monte Carlo procedure to calculate the position distribution of the oscillator, the electrical current, and the zerofrequency noise in both the weak-coupling and strongcoupling regimes. With increasing coupling, we found that the position distribution of the oscillator evolves from a broad Gaussian to a a function sharply peaked around each of the charge-state-dependent equilibrium positions of the oscillator. We found that the average current in the strongcoupling regime is higher than the value predicted by the weak-coupling theory and that the Fano factor varies in a nonmonotonous fashion when coupling is increased. We have generalized the weak-coupling theory to allow the calculation of higher cumulants of the current and have presented results for the third cumulant. In the weak-coupling regime, the third cumulant was found to depend strongly on 
the frequency of the oscillator, whereas in the strongcoupling regime it becomes practically independent of this parameter. We have also studied the frequency-dependent transport noise. Even in the strong-coupling regime, there are no peaks for frequencies higher than $2 \omega_{0}$ and the two peaks found in the weak-coupling limit merge on increasing the coupling strength. Finally, we introduced a generalized expression connecting the damping rate in the strong-coupling regime with the other parameters of our model and used it to understand the evolution of the oscillator's dampingrenormalized frequency as a function of coupling.

\section{ACKNOWLEDGMENTS}

We would like to thank J. Lehmann and M. Vanevic and, particularly, A. D. Armour for interesting discussions and correspondence. This work was financially supported by the Natural Sciences and Engineering Research Council of Canada, the Fonds Québécois de la Recherche sur la Nature et les Technologies, the SFB 513 Nanostructures at Surfaces and Interfaces of the DFG, the Swiss NSF, and the NCCR Nanoscience.

\section{APPENDIX: DETAILS OF THE MONTE CARLO APPROACH USED}

Monte Carlo methods have been used for a long time to calculate numerically the transport properties of mesoscopic systems like SET's. ${ }^{42}$ When dealing with a simple SET system, the idea of the Monte Carlo approach is to solve the master equation for the charge states of the SET by discretizing time into small intervals and allowing charge transfer to and from the dot with a probability that is proportional to the tunneling rates and the time interval between two attempts.
If the SET is coupled to a harmonic oscillator, we can proceed in a similar way by considering charge transfer attempts at a finite frequency $\left(\Delta \tau_{t}\right)^{-1}$, where $\Delta \ll 1$ is a dimensionless step size. The success probability for a charge transfer is calculated from the oscillator's position-dependent instantaneous rates $\Gamma_{i}^{\sigma}(x)$ calculated at the time of the attempt. Between different attempts, the oscillator's state evolves according to the classical equation of motion, whose solution depends on the charge state of the SET, the equilibrium position of the oscillator being shifted by $x_{0}$ when the charge state is changed from $N \rightarrow N+1$ or by $-x_{0}$ in the opposite case. At the beginning of each run, the state of the system is determined randomly from the stationary probability distributions $P_{N}(x, u)$ and $P_{N+1}(x, u)$. In practice, this can be implemented by using the final state of the $(n-1)$ th Monte Carlo run as the initial state of the $n$th run.

We consider runs of total time $t_{0} \tau_{t}$, such that each run consists of $M=t_{0} / \Delta$ steps. Both time scales $t_{0} \tau_{t}$ and $\Delta \tau_{t}$ are chosen in a way such that increasing $t_{0}$ or decreasing $\Delta$ does not affect the value of the different physical quantities we extract from our calculation. In practice, this corresponds to choosing $\Delta<0.02$ and $t_{0} \tau_{t}$ an order of magnitude greater than the typical damping time $1 / \gamma$. A consequence of this last constraint is that the Monte Carlo approach is particularly useful in the strong-coupling regime, where the number of steps, $M$, per run can be kept relatively small, allowing for more runs to be made in the same amount of computer time.

We checked that our code reproduces the analytical results of Ref. 33 for the dependence of the noise and the third cumulant on the asymmetry coefficients $\Delta_{L}-\Delta_{R}$ in the $\kappa=0$ limit. Also, the probability distributions $P_{N}(x, u)$ and $P_{N+1}(x, u)$ that we calculate using the Monte Carlo approach coincide with the results one finds when solving Eq. (9) on a grid. ${ }^{14}$
${ }^{1}$ A. Cleland and M. Roukes, Nature (London) 392, 160 (1998).

${ }^{2}$ M. P. Blencowe, Phys. Rep. 395, 159 (2004).

${ }^{3}$ M. Roukes, Phys. World 14, 25 (2001).

${ }^{4}$ M. LaHaye, O. Buu, B. Camarota, and K. Schwab, Science 304, 74 (2004)

${ }^{5}$ L. Y. Gorelik, A. Isacsson, M. V. Voinova, B. Kasemo, R. I. Shekhter, and M. Jonson, Phys. Rev. Lett. 80, 4526 (1998).

${ }^{6}$ C. Weiss and W. Zwerger, Europhys. Lett. 47, 97 (1999).

${ }^{7}$ A. Erbe, C. Weiss, W. Zwerger, and R. H. Blick, Phys. Rev. Lett. 87, 096106 (2001).

${ }^{8}$ F. Pistolesi, Phys. Rev. B 69, 245409 (2004).

${ }^{9}$ A. Isacsson and T. Nord, Europhys. Lett. 66, 708 (2004).

${ }^{10}$ T. Novotny, A. Donarini, C. Flindt, and A.-P. Jauho, Phys. Rev. Lett. 92, 248302 (2004).

${ }^{11}$ F. Pistolesi and R. Fazio, New J. Phys. 8, 113 (2006).

${ }^{12}$ D. Fedorets, L. Y. Gorelik, R. I. Shekhter, and M. Jonson, Phys. Rev. Lett. 92, 166801 (2004).

${ }^{13}$ D. Fedorets, L. Y. Gorelik, R. I. Shekhter, and M. Jonson, Phys. Rev. Lett. 95, 057203 (2005).

${ }^{14}$ A. D. Armour, M. P. Blencowe, and Y. Zhang, Phys. Rev. B 69, 125313 (2004).
${ }^{15}$ A. D. Armour, Phys. Rev. B 70, 165315 (2004).

${ }^{16}$ D. Mozyrsky, I. Martin, and M. B. Hastings, Phys. Rev. Lett. 92, 018303 (2004).

${ }^{17}$ N. M. Chtchelkatchev, W. Belzig, and C. Bruder, Phys. Rev. B 70, 193305 (2004).

${ }^{18}$ C. Flindt, T. Novotny, and A.-P. Jauho, Europhys. Lett. 69, 475 (2005).

${ }^{19}$ O. Usmani, Y. M. Blanter, and Y. V. Nazarov, cond-mat/0603017 (unpublished).

${ }^{20}$ F. Haupt, F. Cavaliere, R. Fazio, and M. Sassetti, cond-mat/ 0607080 (unpublished).

${ }^{21}$ A. A. Clerk, Phys. Rev. B 70, 245306 (2004).

${ }^{22}$ A. Cleland and M. Roukes, Appl. Phys. Lett. 69, 2653 (1996).

${ }^{23}$ R. G. Knobel and A. N. Cleland, Nature (London) 424, 291 (2003).

${ }^{24}$ S. Sapmaz, Y. M. Blanter, L. Gurevich, and H. S. J. van der Zant, Phys. Rev. B 67, 235414 (2003).

${ }^{25}$ V. Sazonova, Y. Yaish, H. Ustunel, D. Roundy, T. A. Arias, and P. McEuen, Nature (London) 431, 284 (2004).

${ }^{26}$ M. P. Blencowe, Contemp. Phys. 46, 249 (2005).

${ }^{27}$ D. V. Averin, A. N. Korotkov, and K. K. Likharev, Phys. Rev. B 
44, 6199 (1991).

${ }^{28}$ C. W. J. Beenakker, Phys. Rev. B 44, 1646 (1991).

${ }^{29}$ These coefficients were called $\Gamma_{L}$ and $\Gamma_{R}$ in Ref. 14. Since they are not rates (e.g., they can become negative in the strongcoupling limit), we have chosen a different notation.

${ }^{30}$ S. Hershfield, J. H. Davies, P. Hyldgaard, C. J. Stanton, and J. W. Wilkins, Phys. Rev. B 47, 1967 (1993).

${ }^{31}$ A. N. Korotkov, Phys. Rev. B 49, 10381 (1994).

${ }^{32}$ Y. V. Nazarov, Quantum Noise in Mesoscopic Physics (Kluwer, Dordrecht, 2003).

${ }^{33}$ D. A. Bagrets and Y. V. Nazarov, Phys. Rev. B 67, 085316 (2003).

${ }^{34}$ W. Belzig, Phys. Rev. B 71, 161301(R) (2005).

${ }^{35}$ These equations agree with the system of equations shown in the Appendix of Ref. 15; the factor of $k^{2}$ displayed there is a typo and should read $k$.
${ }^{36}$ D. A. Rodrigues and A. D. Armour, Phys. Rev. B 72, 085324 (2005).

${ }^{37}$ Y. M. Blanter and M. Büttiker, Phys. Rep. 336, 1 (2000).

${ }^{38}$ S. Gustavsson, R. Leturcq, B. Simovic, R. Schleser, T. Ihn, P. Studerus, K. Ensslin, D. C. Driscoll, and A. C. Gossard, Phys. Rev. Lett. 96, 076605 (2006).

${ }^{39}$ D. K. C. MacDonald, Noise and Fluctuations: An Introduction (Wiley, New York, 1962).

${ }^{40}$ A. Cottet, W. Belzig, and C. Bruder, Phys. Rev. Lett. 92, 206801 (2004).

${ }^{41}$ A. Cottet, W. Belzig, and C. Bruder, Phys. Rev. B 70, 115315 (2004).

${ }^{42}$ M. Amman, K. Mullen, and E. Ben-Jacob, J. Appl. Phys. 65, 339 (1989). 